\title{
Cytotoxicity and Proteomics Analyses of OSU03013 in Lung Cancer
}

\author{
Yi-Hung Tan, ${ }^{1}$ Kung-Hsueh Lee, ${ }^{1}$ Topp Lin, ${ }^{2}$ Ying-Chieh Sun, ${ }^{2}$ Hsiu Mei Hsieh-Li, ${ }^{1}$ Hsueh-Fen Juan, ${ }^{3}$ \\ and Yi-Ching Wang ${ }^{4}$
}

\begin{abstract}
Purpose: Most lung cancer patients have some resistance to and suffer from side effects of conventional chemotherapy. Thus, identification of a novel anticancer drug with better target selectivity for lung cancer treatment is urgently needed.

Experimental Design: In order to investigate whether OSU03013, a derivative of celecoxib, can be a potential drug for lung cancer treatment, we examined its cytotoxicity mechanisms by flow cytometry and phosphatidylserine staining in A549, CL1-1, and H1435 lung cancer cell lines, which are resistant to the conventional drug, cisplatin. In addition, we identified the affected proteins by proteomics and confirmed the selected proteins by Western blot analysis. We examined the interaction between OSU03013 and potential target protein by molecular modeling. Results: Our results indicated that OSU03013 had low-dose $(1 \sim 4 \mu \mathrm{M})$ cytotoxicity in all lung cancer cell lines tested 48 hours posttreatment. OSU 03013 caused cell cycle G1 phase arrest and showed phosphatidylserine early apoptosis via endoplasmic reticulum stress. Several proteins such as heat shock protein 27,70, and 90, CDC2, $\alpha$-tubulin, annexin A3, cAMP-dependent protein kinase, glycogen synthase kinase 3-beta, and $\beta$-catenin were identified by proteomics and confirmed by Western blot. In addition, molecular modeling showed that OSU03013 competes with ATP to bind to cAMP-dependent protein kinase.

Conclusions: We identified for the first time that OSU03013 inhibits CAMP-dependent protein kinase activity and causes dephosphorylation of glycogen synthase kinase 3-beta leading to $\beta$-catenin degradation, which is often overexpressed in lung cancer. Our molecular and proteomic results show the potential of OSU03013 as an anticancer drug for lung cancer.
\end{abstract}

Over the past decade, lung cancer has been the most common cause of cancer death in the world. Lung cancer causes 1.3 million deaths per year worldwide (1-3). Even with improving efficacy of surgical treatment, radiation therapy, and chemotherapy, poor prognosis for patients with lung cancer remains. Treatment failure, whether from tumor relapse or tumor resistance to chemotherapy often results in metastasis. For this reason, the identification of novel anticancer drugs for lung cancer treatment is urgently needed for cancer therapy.

OSU03013 is a derivative of celecoxib. Although celecoxib is an inhibitor of cyclooxygenase (COX)-2, substantial data indicate that celecoxib-induced apoptosis cell death is through

Authors' Affiliations: Departments of ${ }^{1}$ Life Science and ${ }^{2}$ Chemistry, Nationa Taiwan Normal University, and ${ }^{3}$ Life Science and Institute of Molecular and Cellular Biology, National Taiwan University, Taipei, Taiwan; and ${ }^{4}$ Department of Pharmacology, College of Medicine, National Cheng Kung University, Tainan, Taiwan Received 7/23/07; revised 9/30/07; accepted 10/26/07.

Grant support: Grants NSC96-2628-B-006-048-MY3 and NSC97-2627-M003-002 from the National Science Council (The Executive Yuan, Republic of China) and NTU-PFIR-96R0107 from National Taiwan University.

The costs of publication of this article were defrayed in part by the payment of page charges. This article must therefore be hereby marked advertisement in accordance with 18 U.S.C. Section 1734 solely to indicate this fact.

Note: H.-F. Juan and Y.-C. Wang are equally responsible for this article.

Requests for reprints: Yi-Ching Wang, Department of Pharmacology, College of Medicine, National Cheng Kung University, No. 1, University Rd., Tainan, 70101, Taiwan.Phone: 886-6-2353535; Fax: 886-6-2749296; E-mail: ycw5798@ mail.ncku.edu.tw.

(C) 2008 American Association for Cancer Research.

doi:10.1158/1078-0432.CCR-07-1806 a COX-2-independent pathway (4). A recent study indicated that celecoxib derivatives such as OSU03013 induce apoptosis in prostate cancer cells through the 3-phosphoinositidedependent kinase 1 (PDK1)/AKT signaling pathway and may more strongly inhibit cell growth than celecoxib (5). The docking study showed that OSU03013 can bind to the ATP site of PDK1 leading to its inactivation (5). In addition, OSU03013 has been used in breast cancer treatment, and it has higher cytotoxicity especially in breast cancer cells with epidermal growth factor receptor (HER)-2 overexpression (6).

According to previous studies, OSU03013 can induce cell apoptosis (5-7). Several specific morphologic changes during apoptosis include loss of membrane asymmetry, DNA fragmentation, and nuclear condensation (8). One important signal for phagocytes of apoptotic cells is the exposure of phosphatidylserine (PS). Therefore, PS exposure is crucial for apoptotic cells $(8-10)$. Persistent or strong endoplasmic reticulum (ER) stress can also trigger apoptosis (11). During ER stress, the stress signal induces ER transmembrane protein PRKR-like endoplasmic reticulum kinase (PERK) phosphorylation. Activated phosphor-PERK phosphorylates the eukaryotic translational initiation factor- $2 \alpha$ (eIF2 $\alpha$ ). Phosphor-eIF2 $\alpha$ will then upregulate the protein GADD34 $(11-13)$. Caspase 12 is also activated by ER stress - induced apoptosis (14).

Although OSU03013 has been known to be involved in the AKT signaling pathway and to induce apoptosis in prostate cancer, a recent study by Tong et al. found that $10 \mu \mathrm{M}$ of OSU03013 can induce cytochrome C-mediated apoptosis in A549 lung cancer cells especially at low concentrations of exogenous-expressed AKT (7). 
Therefore, to improve understanding of the molecular mechanisms underlying lung cancer cell treatment with OSU03013 and to investigate its affected molecular targets for lung cancer therapy, the present study examined the cytotoxicity of OSU03013 in lung cancer cells resistant to the conventional anticancer drug, cisplatin, and in a normal lung cell to confirm its selective cytotoxicity to cancer cells. Apoptosis phenotypes of PS staining and ER responses and induction of their related proteins were examined. Two-dimensional electrophoresis (2-DE) was used to reveal the differential proteins affected by OSU03013-treated lung cancer cells, and their expression level was confirmed by Western blot analysis. Our results show for the first time that OSU03013 could affect several pathways such as the cAMP-dependent protein kinase (PKA) and Wnt/ $\beta$ catenin pathways and induce ER stress-induced apoptosis at a dose as low as $2 \mu \mathrm{M}$ in lung cancer cells.

\section{Materials and Methods}

Cell culture. Human normal lung fibroblast cell line MRC5 (American Type Culture Collection, Manassas, VA) and A549 (type II human lung alveolar epithelial cell carcinoma), H1435 lung adenocarcinoma (American Type Culture Collection), and CL1-1 (derived from a Taiwanese female patient with lung adenocarcinoma, which was obtained from Dr. Pan-Chyr Yang, Professor, Department of Internal Medicine, National Taiwan University Hospital, Taipei, Taiwan) cell lines were cultured in DMEM (MRC5, A549, and CL1-1) and RPMI 1640 (H1435) medium (GIBCO, Grand Island, NY) containing 10\% fetal bovine serum (FBS) (BIOCHROM AG, Leonorenstr, Berlin) and 1\% penicillinstreptomycin (GIBCO) and incubated at $37^{\circ} \mathrm{C}$ in $5 \% \mathrm{CO}_{2}$ atmosphere.

Cytotoxicity measurement. $1 \times 10^{5}$ cells/well were seeded in six-well plates and treated with dimethyl sulfoxide (DMSO) and various concentrations of OSU03013 (obtained from Dr. Ching-Shih Chen, Professor, Division of Medicinal Chemistry and Pharmacognosy, College of Pharmacy, The Ohio State University, Columbus, $\mathrm{OH}$ ) containing 10\% FBS for 48 hours, then washed twice with $1 \times$ phosphate-buffered saline (PBS) and stained with Trypan Blue solution $(0.4 \%)$ (Sigma-Aldrich, St. Louis, MO) to measure the effect of OSU03013 on cell growth.

Flow cytometry. Cell cycle distribution was determined by flow cytometry. Approximately $2 \times 10^{6}$ cells were incubated in medium containing $10 \%$ FBS and treated with double IC $_{50}$ of OSU03013 per cell line respectively for 0,24 , and 48 hours. Cells were collected by trypsin/EDTA treatment and washed with ice-cold $1 \times$ PBS three times, then fixed with ice-cold $70 \%$ ethanol overnight. Cells were stained with a solution containing $20 \mu \mathrm{g} / \mathrm{mL}$ propidium iodide, $200 \mu \mathrm{g} / \mathrm{mL}$ RNase A, and $0.1 \%$ Triton X-100 for 15 minutes in the dark. Cell cycle distribution was performed by FACScan flow cytometry (BD Biosciences, Mountain View, CA) and calculated using ModFIT LT 2.0 version software (BD Biosciences).

Early apoptosis detection/phosphatidylserine staining. Cells were seeded at a density of $2 \times 10^{5}$ in $60-\mathrm{mm}^{2}$ dishes. After treatment with DMSO and the respective $\mathrm{IC}_{50}$ concentration of OSU03013 for each cell line for 24 hours, the cells were washed three times in Hanks' Balanced Salt Solution (HBSS) (GIBCO) and incubated with antiphosphatidylserine (PS) primary antibody (Upstate Biotechnology, Lake Placid, NY) for 1 hour at room temperature. Then, the cells were washed with HBSS twice and probed with fluorescent-conjugated secondary antibody (Chemicon International, Temecula, CA) for 1 hour at room temperature. After secondary antibody hybridization, the cells were washed with HBSS twice and then observed with an Olympus BX50 fluorescence microscope (Dulles, VA).

Two-dimensional gel electrophoresis and image analysis and in-gel digestion and matrix-assisted laser desorption/ionization time-of-flight mass spectrometry (MALDI-TOF-MS) and MALDI-TOF-tandem mass spectrometry (MS/MS). CL1-1 or H1435 cell pellets $\left(1 \times 10^{7}\right.$ cells $)$ were solubilized in lysis buffer containing $7 \mathrm{M}$ urea (Boehringer Mannheim, Germany), $2 \mathrm{M}$ thiourea (Sigma-Aldrich, Milwaukee, WI), and 4\% CHAPS (JT Baker, Phillipsburg, NJ). After sonication, $500 \mathrm{mg}$ of total protein was loaded into immobilized pH gradient (IPG) gel strips ( $\mathrm{pH} 3-$ 10 or $\mathrm{pH} 4-7,18 \mathrm{~cm}$ long; Amersham Pharmacia Biotech, Uppsala, Sweden). For the first-dimensional separation, isoelectric focusing was carried out using the IPGphor system (Amersham Pharmacia Biotech) at $20^{\circ} \mathrm{C}$ with $8000 \mathrm{~V}$ for a total of $65 \mathrm{kVh}$ as previously described (15). After isoelectric focusing, the IPG strips were equilibrated for 15 minutes then attached with $0.5 \%$ agarose to the top of a vertical $12.5 \%$ SDSpolyacrylamide gel. Second-dimensional electrophoresis was carried out with Protean II Multi-Cell (Bio-Rad, Hercules, CA) as previously described (15). The gels were fixed and stained in $350 \mathrm{~mL}$ of the Sypro Ruby Protein Gel (Invitrogen, Carlsbad, CA) stain solution overnight, then the residual dye was washed out of the polyacrylamide matrix. The developed gels were digitally scanned as 2-D images using fluorescence image scanning Typhoon 9200, then analyzed using ImageMaster software (both from Amersham Pharmacia Biotech) to automatically detect and quantify protein spots. Intensity levels were normalized between gels as a proportion of the total protein intensity detected for the entire gel. Normalized protein intensity data for matched spots with two-old differences were exported to Gel Report.

From the 2-D gel analysis of DMSO controls and OSU03013-treated samples, differentially expressed proteins were selected for identification by MS and/or MS/MS. These spots were cut from 2-D gels, destained, and dehydrated as previously described (15). Enzyme digestion was performed by adding $15 \mu \mathrm{L}$ trypsin in $25 \mathrm{mM}$ ammonium bicarbonate to a final concentration of $0.0225 \mu \mathrm{g}$ per sample at $37^{\circ} \mathrm{C}$ for 16 hours. The peptide fragments were extracted twice, concentrated, and eluted with $2 \mu \mathrm{L}$ of $100 \%$ acetonitrile and directly spotted on the sample plate of a MALDI-TOF-MS. Finally, $\alpha$-cyano- 4 -hydroxycinnamic acid $(0.5 \mu \mathrm{L}$ of $10 \mathrm{mg} / \mathrm{mL})$ was applied to each spot, and the spots were air-dried at room temperature before acquiring mass spectra (M@LDI; Micromass, Manchester, UK). The resultant peptide masses were matched with the theoretical peptide masses of all proteins from all species in the SWISSPROT and NCBI databases using Masslynx 3.4 software (Micromass). Our protein selection criteria are (1) a match of at least five fragments from a single 2-D gel spot against a single-protein sequence entry in the database, (2) high coverage value, and (3) the sequence is of human origin.

Western blot. Cells were initially seeded at a density of $2 \times 10^{6}$ in $100-\mathrm{mm}^{2}$ dishes. After treatment with DMSO and $\mathrm{IC}_{50}$ concentration of OSU03013 for 48 hours of incubation, the cells were collected and washed twice in $1 \times$ PBS, then lysed in ice-cold lysis buffer $(0.5 \mathrm{M}$ Tris-Hcl with $\mathrm{pH} 7.4,1.5 \mathrm{M} \mathrm{NaCl}, 2.5 \%$ deoxycholic acid, $10 \mathrm{mM}$ EDTA, 10\% NP-40, $0.5 \mathrm{mM}$ DTT, $1 \mathrm{mM}$ phenylmethylsulfonyl fluoride, $5 \mu \mathrm{g} / \mathrm{mL}$ leupeptin, and $10 \mu \mathrm{g} / \mathrm{mL}$ aprotinin) for 5 minutes. The lysate

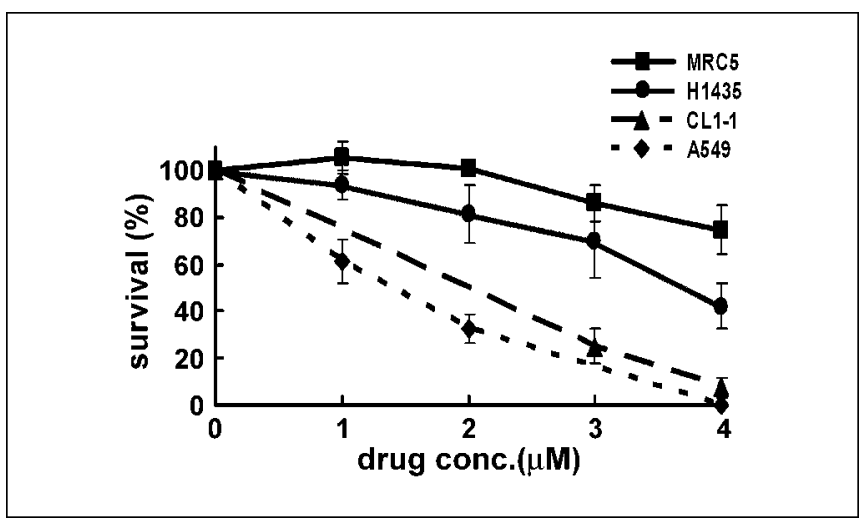

Fig. 1. Cell cytotoxicity of OSU03013 treatment at $48 \mathrm{~h}$. Normal lung fibroblast cell line MRC5 did not show apparent cytotoxicity, whereas lung cancer cell lines H1435, CL1-1, and A549 showed strong killing effect after OSU03013 treatment 
Fig. 2. $A$, flow cytometry analysis of cell cycle distribution of lung cancer cell lines A549, CL1-1, and H1435, treated with their respective $\mathrm{IC}_{50}$ concentration of OSU03013 for 24 and 48 h. G1 arrest was detected as indicated at arrows in three model cell lines. $B$, Western blot analysis of cell cycle checkpoint protein phosphor-CDC2.

Detection showed protein upregulated after treatment with OSU03013. C, Early apoptosis detection using PS staining. After being treated with OSU03013, three cell lines all showed higher PS antibody intensity than untreated cells, as indicated by arrows.

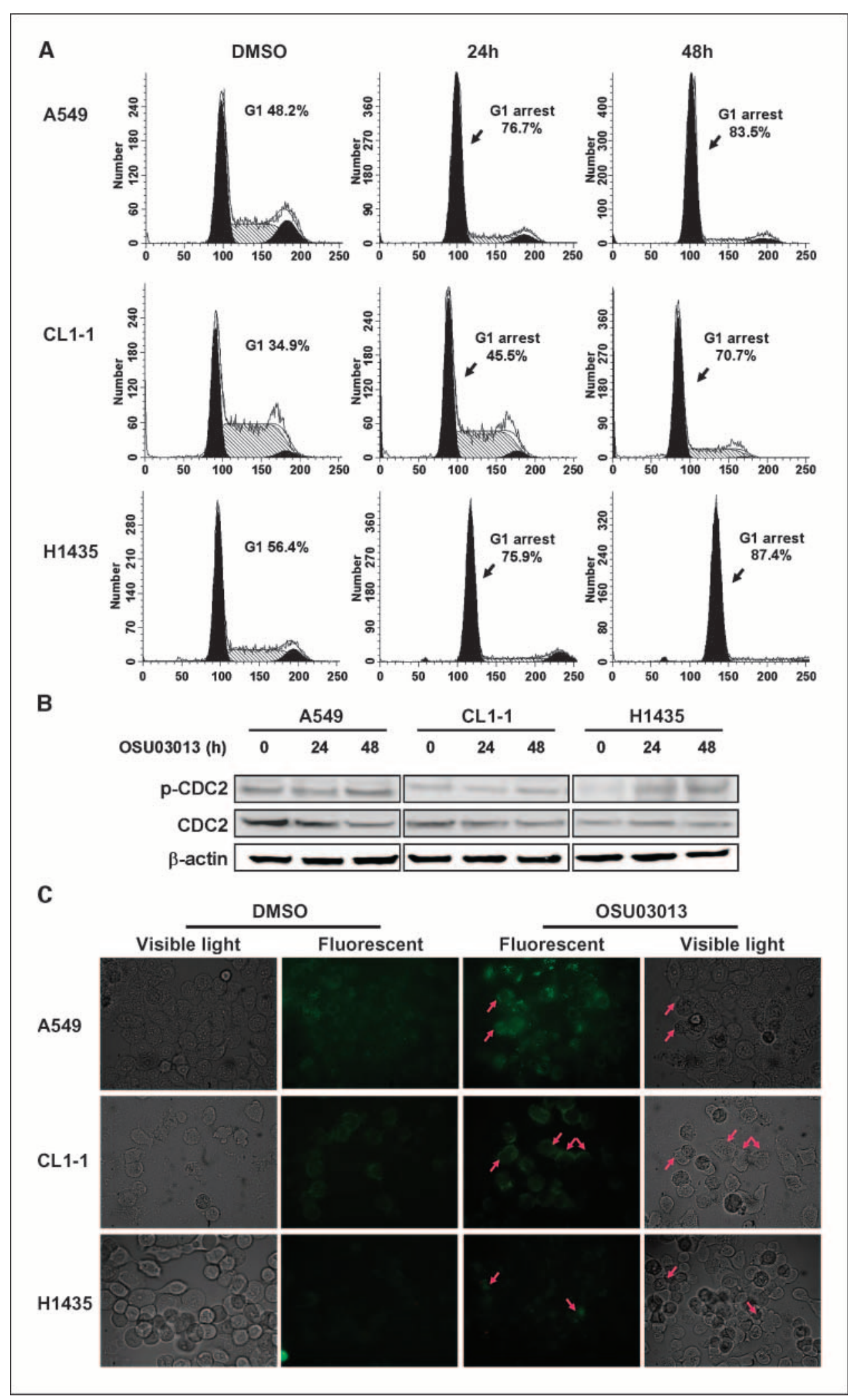

was centrifuged at $13000 \mathrm{rpm}$ for 20 minutes at $4^{\circ} \mathrm{C}$, and protein content of the supernatant was measured. Total cell lysates $(50 \mu \mathrm{g} /$ well $)$ were separated by SDS-PAGE and electrophoretically transferred onto polyvinylidene difluoride membranes (Millipore, Bedford, MA). Membranes were blocked with $5 \%$ skim milk in phosphate-buffered saline tween (PBST) $(1 \times$ PBS, $0.1 \%$ Tween-20) for 1 hour at room temperature and incubated with the appropriate primary antibody (PKA, p-GSK3 $\beta, \beta$-catenin, caspase 12, p-PERK, GADD34, eIF2 $\alpha$, $\alpha$-tubulin, heat-shock protein (Hsp)90, Hsp70, Hsp27, annexin A3, $\mathrm{CDC} 2, \mathrm{p}$-CDC2, $\beta$-actin) at $4^{\circ} \mathrm{C}$ overnight. Membranes then were 


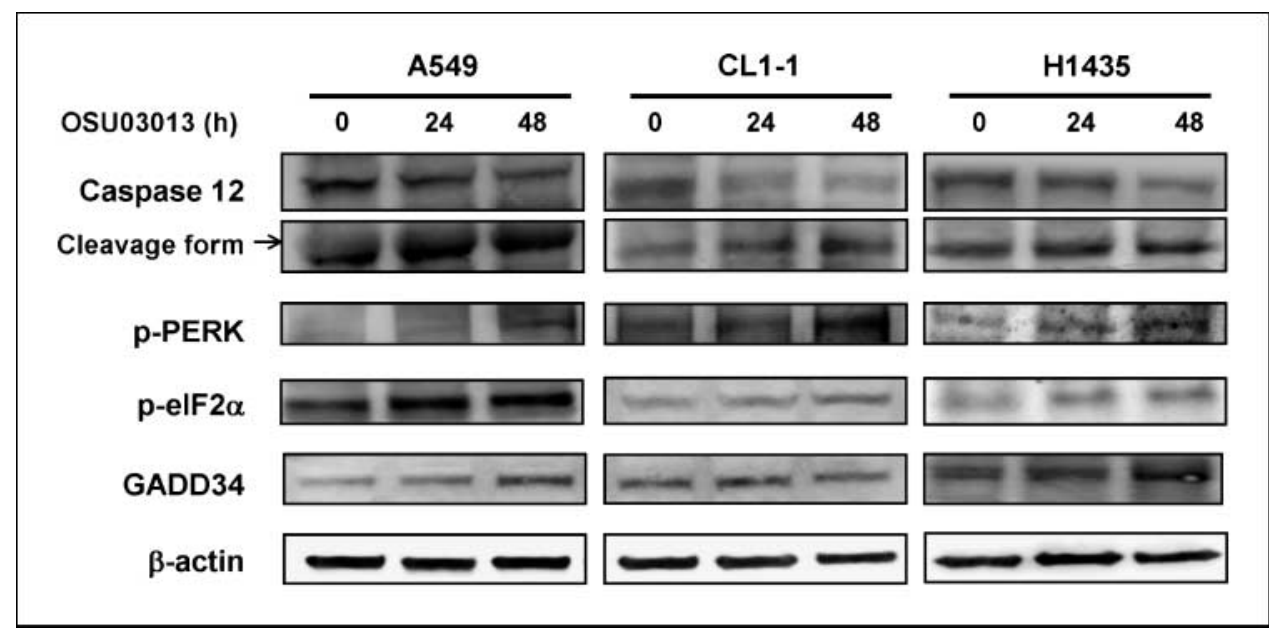

Fig. 3. Western blot analysis of ER stress-related proteins, p-PERK? GADD34, p-elF $2 \alpha$, and caspase-12 were upregulated in most of the lung cancer cells after OSU03013 treatment. washed three times with PBST and probed with appropriate horseradish peroxidase-conjugated secondary antibody for 1 hour at room temperature. After being incubated with the secondary antibody, the membranes were washed three times in PBST. Bands were visualized using Western blot chemiluminescence reagent (Millipore).

Molecular modeling. To examine how OSU03013 interacts with PKA at the molecular level, we carried out docking calculations of OSU03013 to PKA using the Autodock program (16-18). The structure of PKA from the X-ray crystal complex structure (pdb code: 1bx6) (ref. 19) of PKA and bananol, which is an inhibitor of PKA, was used for docking OSU03013. To add hydrogen atoms, the all-atom molecular modeling program, Amber, was used first. The pdb file was then to be treated with Autodock Tools to remove nonpolar hydrogen atoms and keep polar hydrogen atoms only for docking calculation. Atom charges in the protein and the ligand were the default Kollman and GM charges, respectively (17). Atom charges on phosphoserine SEP338 and phosphothreonine TPO197 were obtained from Amber as well. For docking calculation, a docking space of $22.5 \times 22.5 \times 22.5 \AA^{3}$ centered around the ligand binding site (19) was used to ensure sufficient space exploration for ligand docking. Most other docking parameters were set to Autodock default parameters. The number of energy evaluation was set to $2 \times 10^{7}$. This computational protocol was carried out for docking of bananol first, and reproduced the binding mode of balanol-PKA complex (19) with a root mean square deviation of $1.02 \AA$ using the experimental structure as the reference. The same docking protocol was then used for docking OSU03013 to PKA.

\section{Results}

Cytotoxicity of OSU03013 to lung cancer cell lines A549, CL1-1, and H1435, and to normal lung cell line MRC5. To investigate whether OSU03013 showed selective cytotoxicity to cancer cells, we measured the cell growth inhibition of OSU03013 to A549, CL1-1, and H1435 cell lines, which show strong cisplatin resistance, and to the normal lung MRC5 cell line by cell counting with Trypan Blue solution. The $\mathrm{IC}_{50}$ values of A549, CL1-1, and H1435 were $1.53 \mu \mathrm{M}, 1.9 \mu \mathrm{M}$, and $3.5 \mu \mathrm{M}$, respectively after treatment with OSU03013 for 48 hours. Under these concentrations, there was no apparent cytotoxicity to the normal human lung cell line MRC5 (Fig. 1).

OSU03013 induces G1 arrest and early apoptosis. During cytotoxicity study, cell necrosis and apoptosis morphologies were observed. Therefore, we investigated which cell death mechanism was induced by OSU03013 by flow cytometric assay and PS early apoptosis marker staining. After treatment with OSU03013 for 24 and 48 hours, the G1 phase increased to $76.7 \%$ and $83.5 \%$ for A549, $45.5 \%$ and $70.7 \%$ for CL1-1, and $75.9 \%$ and $87.4 \%$ for H1435 (Fig. 2A). Previous reports had shown that phosphorylation at Tyr 15 of CDC2 inhibits the activation of CDC2 and causes cell cycle arrest in G1 phase $(20,21)$. Therefore, we examined phosphor-CDC2 by Western analysis and found that phosphor-CDC2 also increased after OSU03013 treatment in A549 and H1435 cells (Fig. 2B), confirming the observation of G1 arrest. In addition, as cell apoptosis is induced, PS will appear on the cell surface (22). After treatment with OSU03013, three model cells all showed higher PS antibody intensity than untreated cells around the cell membrane, indicating an early apoptosis signal (Fig. 2C).

OSU03013-induced ER stress in lung cancer cell lines A549, CL1-1, and H1435. We examined the activation of caspases-3, 8 , and 9, which are involved in mitochondria and death receptor-associated apoptosis, and the results did not show apparent activation of these caspases (data not shown). Therefore, we speculated that the ER pathway may be involved in the apoptosis induced by low-dose OSU03013. We investigated whether ER stress-induced apoptosis-related proteins such as phosphor-PERK, phosphor-eIF2 $\alpha$, GADD34, and caspase- 12 proteins can be detected in the OSU03013-treated cells. Figure 3 shows that after treatment with OSU03013, phosphor-PERK, phosphor-eIF2 $\alpha$, and GADD34 proteins all increased, and caspase-12 was also cleaved, indicating apoptosis.

Affected proteins of treatment with OSU03013 identified by 2-D electrophoresis/MALDI-Q-TOF. To identify the target and/ or effecter proteins affected by OSU03013, CL1-1, and H1435, cell lines were analyzed by proteomic assays. After treatment with the drug and running with the 2-D electrophoresis (Fig. 4A), we analyzed a total of 207 spots for 2-D/MS and selected 74 differentially expressed proteins with significantly high match scores in the protein database by mass spectrum analyses. Table 1 includes the representative differentially expressed proteins after OSU03013 treatment. To confirm the proteomic results, we performed Western blot analyses on selected proteins. Proteins were selected for further analysis based on both their high match score and their role in signal pathways in cell growth control. Figure $4 \mathrm{~B}$ shows that annexin A3, $\alpha$-tubulin, Hsp27, Hsp70, and Hsp90 proteins were downregulated after treatment with OSU03013. The data coincide with the proteomic results. 
Molecular modeling of OSU03013 to the cAMP-dependent PKA and the activity assay by Western blot of its downstream proteins. According to the proteomic results, cAMP-dependent protein kinase inhibitor beta (PKIB) was upregulated by OSU03013 treatment and may lead to PKA downregulation. Recent studies showed that PKA is an important protein related to cell proliferation (23). Western blot analyses showed that after treatment with OSU03013, PKA was shown to be downregulated, and led to dephosphorylation of glycogen synthase kinase 3-beta (GSK3 $\beta$ ), followed by decreased $\beta$-catenin (Fig. 5A). The docking calculation of OSU03013 to PKA suggested that OSU03013 binds with PKA at the ATP-binding site (Fig. 5B). The phenanthrenyl moiety of OSU03013 is buried in the pocket surrounded by nonpolar residues at the
ATP-binding site. This shows that OSU03013 binding with PKA is mainly stabilized by hydrophobic interaction between phenanthrenyl moiety and the nonpolar residues of PKA at this site. Furthermore, another docking calculation of ATP to PKA (result not shown) showed that the charged phosphate groups of ATP point to the solvent, also supporting that the ATP-binding site favors hydrophobic interaction with ligands. The data support that OSU03013 may compete with ATP for PKA binding and thus inhibit PKA activation.

\section{Discussion}

Our data showed that OSU03013 had selective cytotoxicity against various lung cancer cells but not against the normal lung
Fig. 4. $A$, representative figures of 2-DE electrophotograms of DMSO control and OSU03013 treatment of lung cancer cell line CL1-1. Control (C) represents the untreated cells; CL1-1 treated with OSU03013 is shown as (T). (Upper panel) isoelectric focusing had a $\mathrm{pH}$ range of 4 to 7 , and molecular weight ranged from 21.0 to 97.0 kDa. (Lower panel) Isoelectric focusing ranged from $\mathrm{pH} 3$ to -10 , and the molecular weight ranged from 21.0 to $97.0 \mathrm{kDa}$. Differentially expressed proteins, which circulated in black, were further analyzed, and protein identification was measured by $\mathrm{MS}$ and MS/MS. $B$, Western blot analyses to confirm the results of proteomic experiment are shown. Annexin A3, $\alpha$-tubulin, Hsp27, Hsp70, and Hsp90 proteins were downregulated by treatment with OSU03013 in most of the lung cancer cell lines. The separate images for HSP27 in A549 were due to the individual lanes combined from a single electrophoresis gel. The original gel is shown in the left side of panel B.

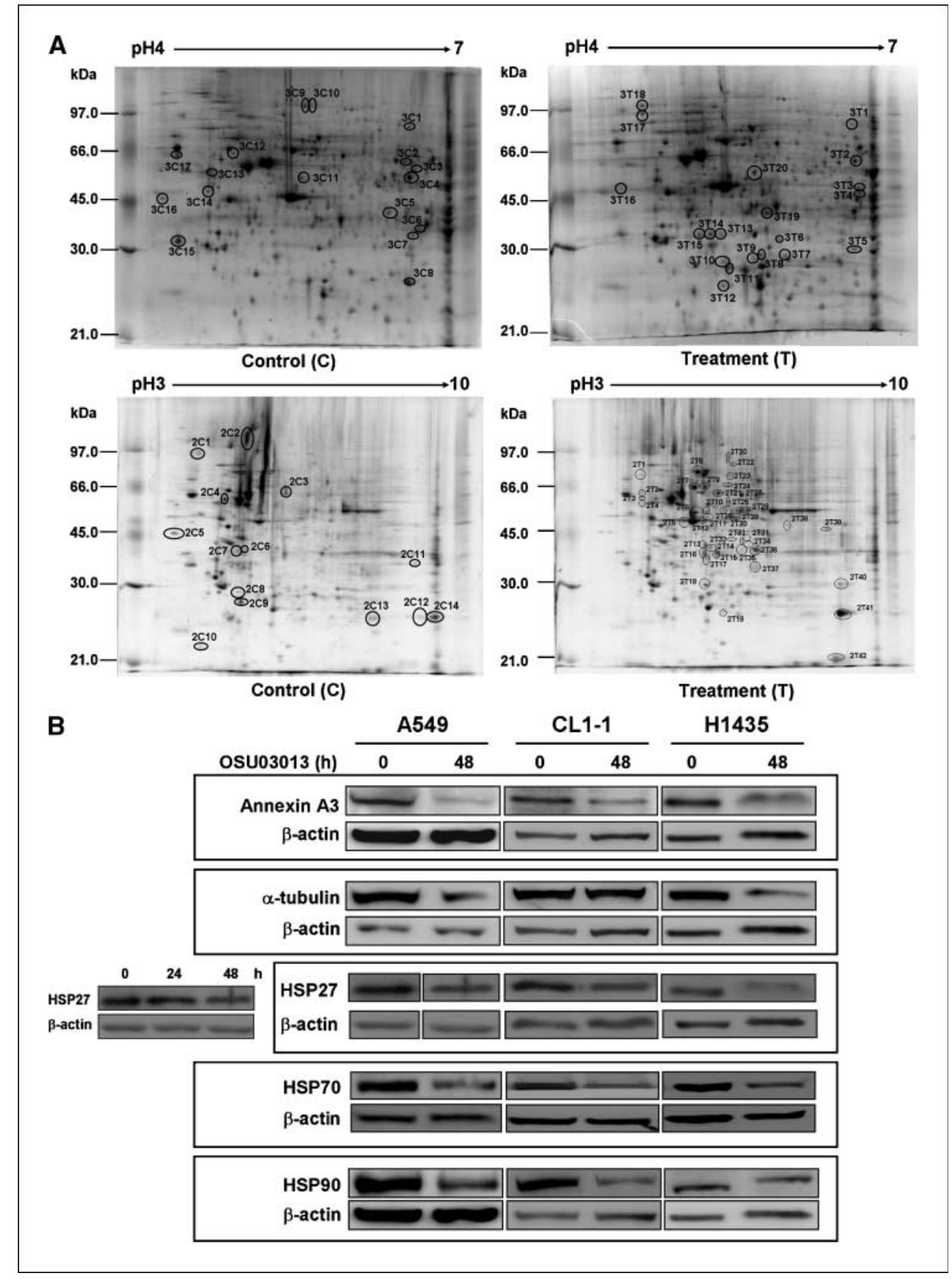


Table 1. MS and MS/MS detection of differential protein spots in lung cancer cells after treatment with OSU03013

\begin{tabular}{|c|c|c|c|c|c|}
\hline Spot* & $\begin{array}{l}\text { Protein } \\
\text { initial name }\end{array}$ & Protein complete name & Accession No. & MW (kDa) & $\begin{array}{l}\text { Propidium } \\
\text { iodide }\end{array}$ \\
\hline \multicolumn{6}{|c|}{ Downregulated proteins } \\
\hline $1 \mathrm{C3}$ & Hsp $90^{\dagger}$ & Heat shock protein HSP 90-beta & $g i \mid 306891$ & 83 & 5.0 \\
\hline $1 C 4$ & $\mathrm{Hsp}^{\dagger} \mathrm{C}^{\dagger}$ & Heat shock 70 kDa protein 8 & $g i \mid 24234686$ & 71 & 5.4 \\
\hline $1 \mathrm{C5}$ & TBA6 & Tubulin alpha- 6 chain & $g i \mid 37492$ & 50 & 5.0 \\
\hline $1 C 9$ & TBB2C & Tubulin beta-2C chain & gi $\mid 5174735$ & 50 & 4.8 \\
\hline $1 \mathrm{C} 10$ & LAMBR & Laminin-binding protein & $g i \mid 34234$ & 32 & 4.8 \\
\hline $1 \mathrm{C} 14 \S$ & $14-3-3 E$ & 14-3-3 protein epsilon & gi|67464424 & 27 & 4.9 \\
\hline $3 C 4 \S$ & EF1G & Translation elongation factor 1 -gamma & gi|4503481 & 50 & 6.3 \\
\hline $3 \mathrm{C} 8 \S$ & PRDX6 & Peroxiredoxin 6 & $g i \mid 3318841$ & 25 & 6.0 \\
\hline $3 C 10^{\ddagger}$ & UBE1 & Ubiquitin-activating enzyme E1 & gi|23510338 & 118 & 5.5 \\
\hline $4 C 2$ & TBB5 & Tubulin beta & gi|18088719 & 50 & 4.8 \\
\hline $4 C 7 \ddagger$ & MACF1 & Microtubule-actin cross-linking factor 1 & gi|55665463 & 505 & 5.2 \\
\hline $4 C 9$ & HSPA9B & Heat shock $70 \mathrm{kDa}$ protein $9 \mathrm{~B}$ precursor & $g i \mid 24234688$ & 74 & 5.9 \\
\hline $4 \mathrm{C} 12 \S$ & $\operatorname{HSP} 27^{\dagger}$ & Heat shock protein 27 (Homo sapiens) & gi|662841 & 22 & 7.8 \\
\hline $4 \mathrm{C} 14$ & PHB & Prohibitin & gi|4505773 & 30 & 5.6 \\
\hline $5 \mathrm{C} 2$ & ANXA3 ${ }^{\dagger}$ & Annexin A3 & gi|1421662 & 36 & 5.6 \\
\hline $6 C 7 \S$ & PRDX4 & Peroxiredoxin 4 & gi|49456297 & 31 & 5.9 \\
\hline $7 C 4 \mp$ & $1433 B$ & 14-3-3 protein beta/alpha & gi|67464627 & 28 & 4.9 \\
\hline 7C9 & GSTP1 & Glutathione S-transferase P & $g i \mid 87564$ & 23 & 5.4 \\
\hline $7 \mathrm{C} 10^{\ddagger}$ & CRABP2 & Cellular retinoic acid-binding protein 2 & $g i \mid 999882$ & 16 & 5.4 \\
\hline $7 \mathrm{C} 16^{\ddagger}$ & MtSSB & Single-stranded DNA-binding protein, mitochondrial & $g i \mid 2624694$ & 15 & 8.2 \\
\hline $10 \mathrm{C} 1$ & LAMBR & Laminin-binding protein & $g i \mid 34234$ & 32 & 4.8 \\
\hline $10 C 3$ & TBA6 & Tubulin alpha- 6 chain & gi|37492 & 50 & 5.0 \\
\hline $10 \mathrm{C} 13$ & PRDX2 & Peroxiredoxin-2 & gi|32189392 & 22 & 5.7 \\
\hline \multicolumn{6}{|c|}{ Upregulated proteins } \\
\hline $1 \mathrm{~T} 3^{\ddagger}$ & $\mathrm{PKIB}^{\dagger}$ & cAMP-dependent protein kinase inhibitor, beta form & gi|55959912 & 9 & 4.8 \\
\hline $1 \mathrm{~T} 8^{\S}$ & EIF4A2 & Eukaryotic translation initiation factor 4AII & $g i \mid 485388$ & 46 & 5.3 \\
\hline $1 \mathrm{~T} 9 \neq$ & $\mathrm{CDC}^{\dagger}$ & Cell division control protein 2 homolog & gi|30582847 & 34 & 8.4 \\
\hline $2 \mathrm{T7}$ & Hsp60 & Chaperonin & $g i \mid 306890$ & 61 & 5.7 \\
\hline $2 \mathrm{~T} 36$ & ANXA1 & Annexin A1 & $g i \mid 442631$ & 35 & 7.8 \\
\hline $2 \mathrm{~T} 41$ & PRDX1 & Peroxiredoxin 1 & gi|4505591 & 22 & 8.3 \\
\hline $3 T 5 \S$ & $\mathrm{GSK} \mathrm{B}^{\dagger}$ & Glycogen synthase kinase 3-beta & $g i \mid 3800883$ & 2 & 3.9 \\
\hline $3 \mathrm{~T} 13^{\ddagger}$ & IP3KA & Inositol 1,4,5-trisphosphate 3-kinase A & gi|124807 & 51 & 7.6 \\
\hline $5 \mathrm{T3}$ & ERp29 & Endoplasmic reticulum protein ERp29 precursor & gi|5803013 & 29 & 6.8 \\
\hline 5T9 & Rab-11 & Ras-related protein Rab-11A & gi|4758984 & 24 & 6.1 \\
\hline $6 \mathrm{~T} 1$ & Rho GDI 1 & Rho GDP dissociation inhibitor & $g i \mid 36038$ & 23 & 5.0 \\
\hline $8 \mathrm{~T} 11^{\ddagger}$ & TRIM28 & Transcription intermediary factor 1-beta & gi|5032179 & 88 & 5.5 \\
\hline
\end{tabular}

*The first number represents the gel analysis (numbers $1-10$ ). The second letter represents decreased spot intensity after drug treatment (C) or increased spot intensity after drug treatment $(T)$. The third number represents the spot number that shows the differential intensity after drug treatment in the gel.

tProteins selected for further Western blot analysis confirmation.

‡Protein only detected in the MS.

§Protein only detected in the MS/MS.

cell. After treatment with $1 \sim 4 \mu \mathrm{M}$ OSU03013 for 24 and 48 hours, lung cancer cell lines showed G1 arrest and early apoptosis signals such as PS exposure and ES stress-related protein upregulation. More then 70 affected proteins were identified by $2-\mathrm{DE} / \mathrm{MS}$, and some were confirmed by Western blot analysis. Molecular docking and activity assay suggested that one of the direct binding targets of OSU03013 in lung cancer cells is PKA. Therefore, OSU03013 may inhibit lung cancer growth through downregulation of the PKA and $\beta$-catenin pathways.

There are three distinct apoptosis signaling pathways: death receptor-, mitochondria- and ER-mediated apoptosis $(8-14,24,25)$. A recent report described treatment with $10 \mu \mathrm{M}$ OSU03013 will induce A549 lung cancer cell apoptosis through the mitochondrial pathway (7). However, the current study using the lowdose treatment of $1 \sim 4 \mu \mathrm{M}$ of OSU03013 did not induce activation of caspase-3, 8, and 9 (data not shown), whereas the ER-specific caspase-12 was evident. Apoptosis induced at low dosage
OSU03013 could occur through ER stress in A549 and other lung cancer cells. In addition, ER stress-associated proteins such as PERK, eIF2 $\alpha$, and GADD34 were upregulated after drug treatment. Therefore, our data suggest that low-dose OSU03013 may induce cell apoptosis via the ER stress pathway, at least in the lung cell model.

Our study is the first using proteomics to identify the proteins affected by OSU03013. These differentially expressed proteins include cell cycle-related proteins (e.g., $\alpha$-tubulin and CDC2), Hsps (e.g., Hsp27, Hsp70, and Hsp90), and the inositol phosphate metabolism enzyme, annexin A3. Most importantly, using proteomics and molecular modeling, we found that OSU03013 reduced the PKA signaling pathway by upregulating PKIB and leading to low expression of $\beta$-catenin. The PKA pathway has been shown to be connected to the Wnt/ $\beta$-catenin pathway (22). PKA overphosphorylation results in GSK3 $\beta$ overphosphorylation, which is a protein involved 
in the $\beta$-catenin degradation complex. Phosphor-GSK3 $\beta$ causes $\beta$-catenin release from an AXIN/APC degradation complex and results in $\beta$-catenin accumulation, which then translocates to the nucleus to become a transcription factor. Furthermore, overexpression of $\beta$-catenin had been reported in lung cancer $(26,27)$. These results show the potential of OSU03013 as an anticancer drug for lung cancer.

Recent reports indicate that PKA phosphorylation is important for multiple cell functions such as cell proliferation, differentiation, development, metabolism, ion channel activity,

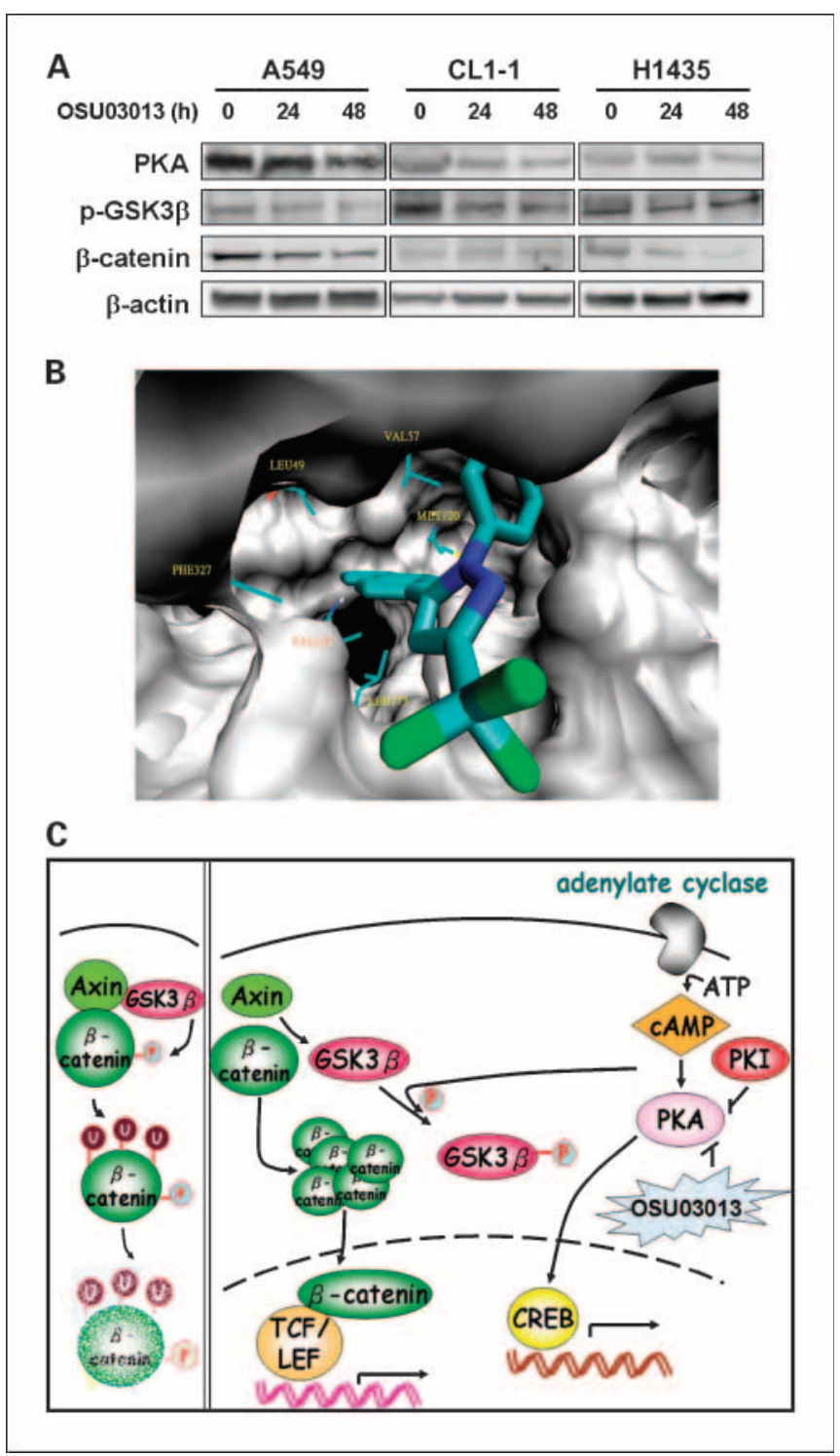

Fig. 5. $A, \mathrm{PKA}$ and GSK $3 \beta$ pathway. Western blot analysis shows PKA dephosphorylation leading to dephosphorylation of GSK3 $\beta$ and degradation of the $\beta$-catenin pathway after OSU03013 treatment. $B$, docking study of OSU03013 compound against PKA. PKA protein surface (in gray) and selected residues at the binding site of OSU03013 are shown in color by means of theAutodock docking program. Data indicate that the compound OSU03013 is located as a ligand at the ATP binding site. $C$, the PKA signaling pathway model. The cell survival signal passes through a ligand on the cell membrane and induces PKA phosphorylation and phosphated GSK $3 \beta$ by phosphor-PKA. Therefore, the complex of GSK3 $\beta$, Axin, and $\beta$-catenin in the cytoplasm regulate cell proliferation. However, OSU03013 can inhibit PKA activity and result in the dephosphorylation of GSK3 $\beta$. This dephosphorylated GSK3 $\beta$ increases its function on $\beta$-catenin degradation and results in cancer cell growth inhibition. (Panel $\mathrm{C}$ is modified from ref. 37 .) and synaptic transmission $(28,29)$. In addition, PKA is involved in the tobacco nitrosamine 4-(methylnitrosamino)-1-(3-pyridyl)-1-butanone (NNK)-induced ERK1/2 and CREB/ATF-1 phosphorylation in pulmonary adenocarcinoma cells and airway epithelial cells, supported by the study using a PKA inhibitor, which partially inhibited NNK-induced DNA synthesis and ERK1/2 activation in these cells (30). The regulatory role of CAMP/PKA signaling is also evident in a cooperation between NNK-induced adrenergic receptors $\beta 1$ and estrogen receptor $\beta 1$ mitogenic signaling in the cell type originating from lung adenocarcinoma (31). In addition, increased intracellular second message cAMP following adenylyl cyclase stimulation activates PKA phosphorylation, and phosphor-PKA phosphorylates downstream cAMP response element-binding protein (CREB) to induce expression of several genes such as c-Jun, StAR, etc. (Fig. 5C; refs. 28, 32). Therefore, whether PKA reactivation or adenylyl cyclase overexpression will reduce OSU03013 cytotoxicity, or whether adenylyl cyclase inhibition or cAMP inhibitors will augment OSU03013 efficiency are worthy of future investigation.

Furthermore, molecular modeling data showing the interaction between OSU03013 and PKA indicate that OSU03013 inhibits PKA by competing with ATP for binding. We proposed that PKA activity can be inhibited by OSU03013 because OSU03013 competes with ATP for PKA binding. This inactivated PKA is thus less able to phosphorylate its downstream proteins such as GSK3 $\beta$. We are now conducting more biochemical and biophysical experiments to confirm the OSU03013PKA interaction to prove that one of the direct targets of OSU03013 is PKA. In addition, CREB will be examined for its expression level and activity to verify the effects on the PKA signaling pathway after OSU03013 treatment. We speculate that other PKA inhibitors such as H89 and KT5720 $(33,34)$ may also have potential as new candidate drugs for lung cancer treatment.

The proteomic study showed that OSU03013 is a multifactorial drug. It downregulated several Hsps including Hsp27, Hsp70, and Hsp90, which will inhibit cell apoptosis and differentiation $(35,36)$. In addition, OSU03013 downregulated annexin A3, which is an enzyme involved in phosphatidyl inositol metabolism. Furthermore, the results of proteomics indicated that cell cycle-related proteins such as $\alpha$-tubulin and phosphor-CDC2 were downregulated and upregulated by OSU03013 treatment, respectively. CDC2 is a G2/M phase checkpoint protein. However, once CDC2 is phosphorylated at Tyr 15, it will inhibit CDC2 activation and cause cell cycle arrest in the G1 phase. These data are consistent with our flow cytometry analysis.

\section{Conclusion}

In conclusion, we identified for the first time that OSU03013 inhibited PKA activity and caused dephosphorylation of GSK3 $\beta$, leading to $\beta$-catenin degradation, which is often overexpressed in lung cancer. Our proteomic and molecular results showed the potential of OSU03013 to be a novel anticancer drug in many lung cancer cells even at low dosage.

\section{Acknowledgments}

We thank Dr. Ching-Shih Chen at the Division of Medicinal Chemistry and Pharmacognosy, College of Pharmacy, The Ohio State University, Columbus, $\mathrm{OH}$, for kindly providing the OSU03013 compound. 


\section{References}

1. World Health Organization: programmes and projects: media center: fact sheets; Feb, 2006 http:// www.who.int/mediacentre/factsheets/fs297/en/.

2. Department of Health, The ExecutiveYuan, Republic of China. R. O. C. Press, Taipei 2007, General Health Statistics, 2006. In: Health and Vital Statistics, Republic of China. http://www.doh.gov.tw/statistic.

3. Travis WD, Lubin J, Ries L, Devesa S. United States lung carcinoma incidence trends: declining for most histologic types among males, increasing among females. Cancer 1996:77:2464-70.

4. Zhu J, Song X, Lin HP, et al. Using cyclooxygenase-2 inhibitors as molecular platforms to develop a new class of apoptosis-inducing agents. J Natl Cancer Inst 2002;94:1745-57.

5. Zhu J, Huang JW, Tseng PH, et al. From the cyclooxygenase-2 inhibitor celecoxib to a novel class of 3 -phosphoinositide-dependent protein kinase-1 inhibitors. Cancer Res 2004;64:4309-18.

6. Kucab JE, Lee C, Chen CS, et al. Celecoxib analogues disrupt Akt signaling, which is commonly activated in primary breast tumours. Breast Cancer Re 2005;7:R796-807

7. Tong Z, Wu X, Chen CS, Kehrer JP. Cytotoxicity of a non-cyclooxygenase-2 inhibitory derivative of celecoxib in non-small-cell lung cancer A549 cells. Lung Cancer 2006;52:117-24.

8. Munoz LE, Frey $B$, Pausch $F$, et al. The role of annexin A5 in the modulation of the immune response against dying and dead cells. Curr Med Chem 2007;14:271 -7.

9. Hirt UA, Leist M. Rapid, noninflammatory and PS dependent phagocytic clearance of necrotic cells. Cell Death Differ 2003:10:1156-64.

10. Hoffmann PR, deCathelineau AM, Ogden CA, et al. Phosphatidylserine (PS) induces PS receptor-mediated macropinocytosis and promotes clearance of apoptotic cells. J Cell Biol 2001:155:649-59.

11. Boyce M, Yuan J. Cellular response to endoplasmic reticulum stress: a matter of life or death. Cell Death Differ 2006:13:363-73

12. Boyce $M$, Bryant KF, Jousse $C$, et al. A selective in hibitor of elF2alpha dephosphorylation protects cells from ER stress. Science 2005;307:935-9.

13. Kojima E, Takeuchi A, Haneda M, et al. The function of GADD34 is a recovery from a shutoff of protein synthesis induced by ER stress: elucidation by GADD34-deficient mice. FASEB J 2003;17:1573-5.
14. NakagawaT, Zhu H, Morishima N, et al. Caspase-12 mediates endoplasmic-reticulum-specific apoptosis and cytotoxicity by amyloid-beta. Nature 2000;403: 98-103.

15. Juan HF, Lin JY, Chang WH, et al. Biomic study of human myeloid leukemia cells differentiation to macrophages using DNA array, proteomic, and bioinformatic analytical methods. Electrophoresis 2002:23. 2490-504

16. Goodsell DS, Olson AJ. Automated docking of substrates to proteins by simulated annealing. Proteins 1990;8:195-202.

17. Morris GM Goodsell DS, Halliday RS, et al Automated docking using a Lamarckian genetic algorithm and an empirical binding free energy function. J Comput Chem 1998:19:1639-62

18. Morris GM, Goodsell DS, Huey R, Olson AJ. Distributed automated docking of flexible ligands to proteins: Parallel applications of AutoDock 2.4. J Comput Aided Mol Des 1996:10:293-304.

19. Narayana N, Diller TC, Koide K, et al. Crystal structure of the potent natural product inhibitor balanol in complex with the catalytic subunit of cAMPdependent protein kinase. Biochemistry 1999;38: 2367-76.

20. HunterT. Protein kinases and phosphatases: the yin and yang of protein phosphorylation and signaling. Cell 1995:80:225-36.

21. Watanabe N, Broome M, HunterT. Regulation of the human WEE1Hu CDK tyrosine 15-kinase during the cell cycle. EMBO J 1995:14:1878-91.

22. Williamson $P$, Schlegel RA. Hide and seek: the secret identity of the phosphatidylserine receptor. Jiol 2004;3:14

23. Masai I, Yamaguchi $M$, Tonou-Fujimori $N$, Komori A, Okamoto $\mathrm{H}$. The hedgehog-PKA pathway regulates two distinct steps of the differentiation of retinal ganglion cells: the cell-cycle exit of retinoblasts and their neuronal maturation. Development 2005; $132: 1539-53$.

24. Mehmet $\mathrm{H}$. Caspases find a new place to hide. Nature 2000:403:29-30.

25. Szegezdi E, Fitzgerald U, Samali A. Caspase-12 and ER-stress-mediated apoptosis: the story so far. Ann N YAcad Sci 2003:1010:186-94

26. Mazieres J, He B, You L, Xu Z, Jablons DM. Wnt sig naling in lung cancer. Cancer Lett 2005;222:1 -10.
27. Uematsu K, He B, You L, Xu Z, McCormick F, Jablons DM. Activation of the Wnt pathway in non small cell lung cancer: evidence of dishevelled overexpression. Oncogene 2003;22:7218-21.

28. Feliciello A, Gottesman ME, Avvedimento EV cAMP-PKA signaling to the mitochondria: protein scaffolds, mRNA and phosphatases. Cell Signal 2005:17:279-87.

29. Montminy M. Transcriptional regulation by cyclic AMP. Annu Rev Biochem 1997;66:807-22.

30. Laag E, Majidi M, Cekanova M, Masi T, Takahashi T, Schuller HM. NNK activates ERK1/2 and CREB/ATF-1 via beta-1-AR and EGFR signaling in human lung adenocarcinoma and small airway epithelial cells. Int J Cancer 2006;119:1547-52.

31. Majidi M, Al-Wadei HA, Takahashi T, Schuller HM Nongenomic beta estrogen receptors enhance beta1 adrenergic signaling induced by the nicotine-derived carcinogen 4- (methylnitrosamino) -1-(3-pyridyl) 1 -butanone in human small airway epithelial cells. Cancer Res 2007;67:6863-71.

32. Stocco DM, Wang $X$, JoY, Manna PR. Multiple sig naling pathways regulating steroidogenesis and steroidogenic acute regulatory protein expression: more complicated than we thought. Mol Endocrinol 2005 19.2647-59

33. Marunaka Y, Niisato N. H89, an inhibitor of protein kinase A (PKA), stimulates $\mathrm{Na}+$ transport by translocating an epithelial $\mathrm{Na}+$ channel $(\mathrm{ENaC})$ in fetal rat alveolar type II epithelium. Biochem Pharmacol 2003; 66:1083-9.

34. Olsen MK, Reszka AA, Abraham I. KT5720 and U-98017 inhibit MAPK and alter the cytoskeleton and cell morphology. J Cell Physiol 1998;176: 525-36

35. Xu W, Neckers L. Targeting the molecular chaperone heat shock protein 90 provides a multifaceted effect on diverse cell signaling pathways of cancer cells. Clin Cancer Res 2007;13:1625-9.

36. Garrido C, Brunet M, Didelot $C$, Zermati Y, Schmitt E, Kroemer G. Heat shock proteins 27 and 70: antiapoptotic proteins with tumorigenic properties. Cell Cycle 2006:5:2592-601.

37. Castellone MD, Teramoto H, Williams BO, Druey KM, Gutkind JS. Prostaglandin E2 promotes colon cancer cell growth through a Gs-axin-beta-catenin signaling axis. Science 2005;310:1504-10. 